\title{
Flowcuits: Crafting Tangible and Interactive Electrical Components with Liquid Metal Circuits
}

\author{
Yutaka Tokuda* \\ Department of Informatics, The \\ University of Sussex \\ Brighton, United Kingdom \\ yutakamitsue@gmail.com
}

\author{
Deepak Ranjan Sahoo \\ Department of Computer Science, \\ Swansea University \\ Swansea, United Kingdom \\ d.r.sahoo@swansea.ac.uk
}

\author{
Matt Jones \\ Department of Computer Science, \\ Swansea University \\ Swansea, United Kingdom \\ Matt.Jones@swansea.ac.uk
}

\author{
Sriram Subramanian \\ UCL Interaction Centre, University \\ College London \\ London, United Kingdom \\ s.subramanian@ucl.ac.uk
}

\begin{abstract}
We present Flowcuits, a DIY fabrication method to prototype tangible, interactive and functional electrical components by manipulating liquid metals. The prototypes afford both physical and visual interactions to demonstrate the inner working mechanics of fundamental electronic elements, which enables tangible and playful learning. The fabrication process follows simple imprinting and sealing of fluidic circuits with a 3D-printed stamp on an accessible moldable-substrates such as 'Blu Tack'. Utilizing conductive gallium indium liquid metal, we demonstrated interactive and reconfigurable electronic components such as switches, variable resistors, variable capacitors, logic gates and pressure sensors. In this paper, we present the design analogy of Flowcuits, DIY fabrication approach including a parametric 3D stamp design toolkit and results from a technical evaluation. The stamps are printed with a low-cost 3D printer and all the materials are inexpensive and reusable, enabling Flowcuits to be easily used without any advanced lab facilities.
\end{abstract}

\section{CCS CONCEPTS}

- Human-centered computing $\rightarrow$ Ubiquitous and mobile computing; User interface toolkits; Human computer interaction (HCI).

\section{KEYWORDS}

Liquid Metal; Liquid Electronics; Fluidics; Playful Learning; Physical Computing; DIY; 3D-printed Stamp; Reusable Material

\section{ACM Reference Format:}

Yutaka Tokuda, Deepak Ranjan Sahoo, Matt Jones, Sriram Subramanian, and Anusha Withana. 2021. Flowcuits: Crafting Tangible and Interactive

*Both authors contributed equally to the paper

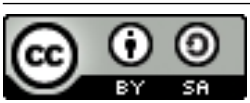

This work is licensed under a Creative Commons Attribution-ShareAlike International 4.0 License.

TEI '21, February 14-17, 2021, Salzburg,

Austria . ACM ISBN 978-1-4503-8213-7/21/02

https://doi.org/10.1145/3430524.3440654
Anusha Withana*

School of Computer Science and

Sydney Nano Institute (Sydney Nano),

The University of Sydney

Sydney, NSW, Australia

anusha.withana@sydney.edu.au

Electrical Components with Liquid Metal Circuits. In Fifteenth International Conference on Tangible, Embedded, and Embodied Interaction (TEI '21), February 14-17, 2021, Salzburg, Austria. ACM, New York, NY, USA, 11 pages. https://doi.org/10.1145/3430524.3440654

\section{INTRODUCTION}

Tangible computing encourages exploration, engagement and reflection in the context of playful learning $[43,44]$. There are many physical computing toolkits designed to teach complex ideas to laypeople leveraging these positive characteristics $[3,8,21,31,37,46]$. Particularly, several do-it-yourself (DIY) toolkits available to easily learn and explore electronic designs through assembling and modifying modular components $[9,20]$. Commercially available open-source platforms such as Arduino, Teensy and Raspberry Pi also promote playful learning.

While these methods successfully combine pre-made electronic components, at the very fundamental level, for instance explaining the functionality of a resistor, a capacitor or a simple sensor, these approaches are less effective. However, such basic principles are crucial in the learning processes and teachers often use simple props such as resistors made out of ionic solution (e.g., salt water, lemon juice) to explain such concepts. However, a method to easily craft and explore the operation of such fundamental components is currently unavailable.

In this paper, we introduce Flowcuits, an extensible, modular and DIY approach to prototype and interactively explore tangible electronic components, circuits and their operations. We use malleable molding substrates such as clay into visual and tangible forms, a familiar crafting approach, as the means of prototyping electronics (Figure 1). Compared to previous approaches, the proposed method can create conductive fluidic circuits on accessible substrates such as "Blu Tack" using modular 3D stamps without any advanced lab facilities [10, 11, 28, 34, 42, 45, 53, 58]. Utilizing liquid-metal and other electrolytic fluids (e.g., $\mathrm{NaOH}$ ) as the active material, we demonstrate the possibility of creating a variety of components such as switches, resistors, capacitors; then extend them to create complex interactive elements such as physical sensors and logic gates. In Flowcuits, we follow a tangible-visual-electrical analogy, which we believe helps broaden the understanding of the inner working of 


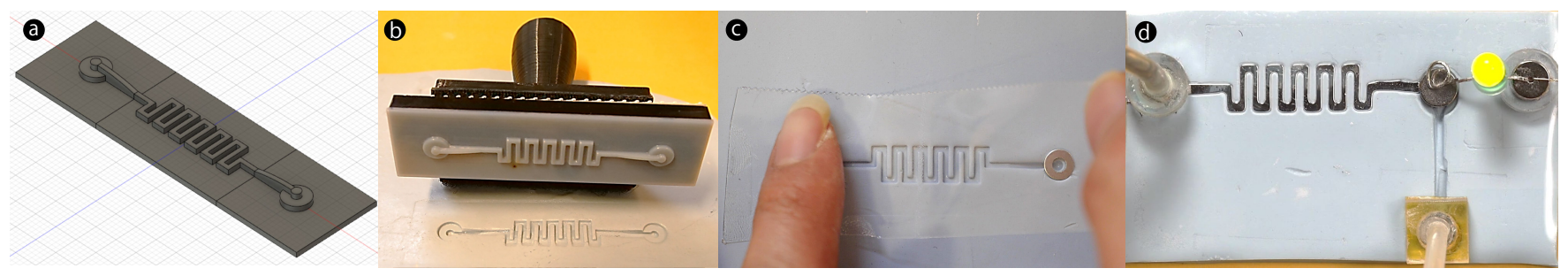

Figure 1: Flowcuits: A DIY Toolkit of Tangible Liquid Electronics: (a) Design a circuit stamp with Flowcuits Stamp CAD toolkit by combining a set of modular stamp elements. (b) 3D print the designed stamp and press it on a flat molding substrate. (c) Seal the fluid channel with a clear adhesive tape and create fluid I/O ports (d) Infuse conductive liquids into the channel to create a liquid electrical circuit by infusing one or more conductive liquids with different resistivity. Then, the user can interactively probe how the brightness of LED changes to explore the principle of resistivity.

fundamental electronic elements utilizing familiar knowledge with unfamiliar and novel [16]. The main contributions of this paper are:

- An easy to use and accessible fabrication approach and a parametric design tool for prototyping fluidic electronic components using reusable and affordable materials in a DIY setting without advanced lab facilities.

- An interactive tangible-visual-electrical analogy to model and explore fundamental electrical concepts and elements.

- A technical evaluation of basic principles of the concept and a set of example applications.

\section{RELATED WORK}

In alignment with our contributions, we will discuss literature with respect to the importance of tangible computing in playful learning, fluidic devices and their applications, and fabrication approaches for fluidic devices.

\subsection{Physical Computing for Playful Learning}

Price et. al. proposed that playful learning through tangible computing enables exploration through interaction, engagement, reflection, imagination, creativity and collaboration [44]. There are many physical computing toolkits that enable tangible interactions for learning $[8,43]$. In the context of this paper, there are several toolkits designed to learn electronics through physical prototyping [3]. Most commonly used approach is programming embedded systems through physical interactions [31, 32, 46]. Also, there are modular toolkits that help learning process through interconnecting pre-built components that help design physical widgets [13], new circuit design techniques [21,37], introduce creativity to new audiences [47], explore new materials [26], and provide augmented learning opportunities [5].

However, there is a gap in teaching the most fundamental concepts of electronics such as inner working of circuit components; for instance, how a capacitor works? This is because toolkits rely on off-the-shelf pre-made components. Educational research identifies teaching the underlying principles of science and technology as one of the biggest challenges and the available tools to support teaching conceptual learning remain under-explored [14, 39]. DIY playful learning materials such as Squishy Circuits try to address these educational problems, but the capabilities are limited in the scope to galvanic contact and resistance [20]. In Flowcuits, we approach this problem by introducing a rapid and accessible prototyping method to build a variety of fundamental electronic components using liquid metal circuits along with hands-on interactions to explore and manipulate the functionality.

\subsection{Fluidic Devices}

Unique properties of liquid materials have been exploited in fluidic devices to create diverse applications such as sensing input [42, 49$51,54,58]$, visual and haptic output [15, 18, 27, 48, 55], and soft robotics [2, 11, 22, 30, 53,57]. Recent advances in the use of fluidics in human-computer interaction explore the interactive capabilities of fluidic mechanisms [34] and shape-changing materials [28]. In Flowcuits, we aim to use conductive fluids as the functional element to combine visual and physical interactions with electrical changes.

Liquid metal, in particular, has gained attention as a configurable material to develop small electro-mechanical systems based on the electrowetting effect, such as a micro-mirror optoelectronic device [56]. Zeng et al. demonstrated a MEMS tilting-mirror made of a liquid metal droplet which was actuated like a torsional spring through electrostatic torque [60]. Huh et al. utilized a conductive liquid metal droplet as a flexible electronic contact to build a simple resistive dual-axis accelerometer with improved longevity [17]. Since the high surface tension of liquid metal allows the reversible deformation of a conductive body, it was exploited to reconfigure the bandwidth of RF antenna [23]. Niiyama et al. utilized high density and fluidness of liquid metal to create a mass-volume controllable device based on a hydraulic pumping system [38]. Fassler and Majidi demonstrated strain sensors using capacitive and inductive fluidic elements [10]. We employed a similar mechanism as previous works $[10,11,42,45,58]$ for infusing liquid metal to prototype interactive liquid electronics. Main advantage of Flowcuits is the simple and DIY fabrication process along with the analogical interactive approach which can be easily replicated without any advanced lab facilities.

\subsection{Fabrication of Liquid Metal Devices}

Researchers have proposed many advanced fabrication approaches to develop liquid metal devices for microfluidic electronics, such as a soft lithography for micro-level patterning [24]. Since the aim of 

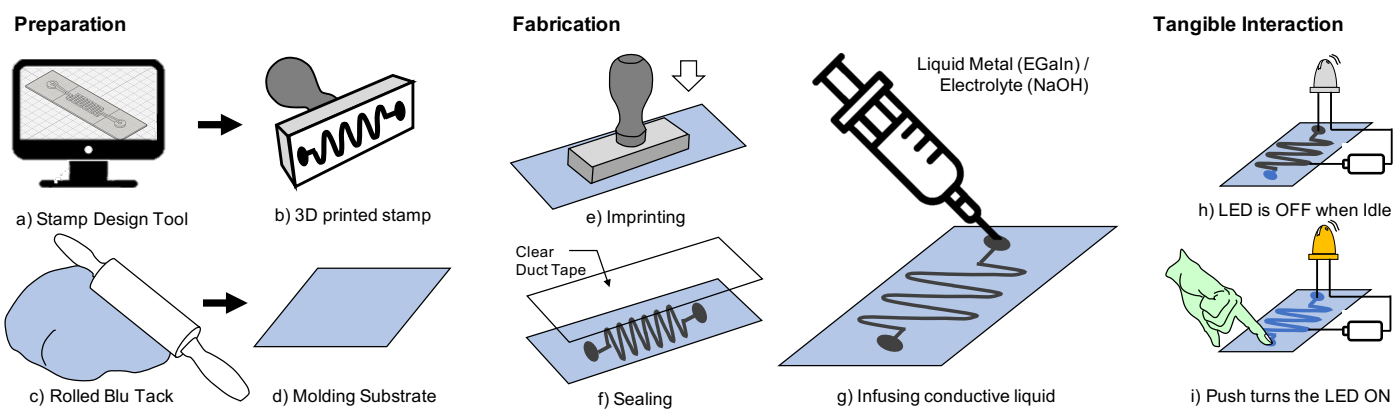

Figure 2: Flowcuits workflow. a) Design tool helps to create fluid circuit stamp; b) 3D print or laser cut the physical stamp; c,d) prepare the flat malleable substrate, e) use the stamp to imprint the pattern, f) shield with transparent tape, f) infuse appropriate liquid conductor, $h, i)$ create tangible interactive circuits

this paper is to facilitate the development of liquid metal devices for tangible playful learning, we focus the scope of this subsection on DIY fabrication approaches. Jeong et al., for instance, proposed liquid metal alloy printing techniques using a stencil mask created by a vinyl-cutter and printed liquid metal along the masked pattern onto a semi-cured polydimethylsiloxane (PDMS) substrate [19]. Direct laser patterning of a conductive-PDMS substrate without a stencil mask was also proposed to accelerate the rapid prototyping of soft-matter electronics [29]. Likewise, Silicone Devices introduced laser engraving, laser patterning and vinyl masking techniques in a scalable DIY fabrication approach for soft-matter circuits [36]. Whereas, Morgan et al. explored 3D printing techniques as alternative fabrication methods of microfluidic devices [35]. While these techniques are effective to make complex and dense circuit traces on soft materials, the development processes require relatively advance facilities and lack incremental learning opportunities through tangible exploration and hands-on discovery.

Conversely, Montgomery et al. proposed a simple stamping method to fabricate a fluid channel on a PDMS substrate [33]. This approach allows more freedom to change designs and supports trial-errors in the middle of development process. However, once PDMS is cured, the creator must restart a fabrication process from scratch to modify the design. Although we follow a similar approach, we extend the strength of trial-errors in stamping process by deploying reusable soft materials that can be quickly molded and reformed to enable hands-on exploration and modification of the design without concerning curing time. Notably, we focus on the fabrication process based on hobby-craft molding materials like clay, so that anyone can easily transfer their knowledge and experience of stamping and handcraft to the workflow of Flowcuits.

\section{FLOWCUITS}

Flowcuits is a simple DIY toolkit inspired by popular crafting technique used with soft and malleable substrates, that we reappropriate for hands-on prototyping electronic components and circuits. The main goal of the proposed method is to facilitate the tangible and visual exploitation of fundamental concepts of electricity through free exploration. We believe this approach could promote creativity and engagement in learning processes through interactive visualization of the inner working mechanisms and familiar tangible interactions for play (e.g., Play-Doh). This section introduces the Flowcuits workflow, the interactive analogy and the intended application directions.

\subsection{Flowcuits Workflow}

Flowcuits workflow can be explained in three simple steps: 1) design and preparation, 2) fabrication, and 3) tangible interactions to explore functionality (outlined in Figure 2).

Design and preparation: Design of the stamp plays a key role in determining which components or circuits will be embossed. We introduce a 3D CAD toolkit which can be used to easily design functional elements of liquid electronic devices and export 3D printable models as custom stamping dies (more details on the tool in Section 4.1). Stamps follow a modular design and do not need to be printed or adjusted for each circuit. Therefore, in real application settings, basic stamps can be pre-built and re-used.

Substrate material also needs a preparation step since most of the crafting materials need to be flattened to create thin substrates required for the smooth stamping process. In our applications, we either used a manual roller or a rolling machine (Figure 2-c,d).

Fabrication: Fabrication process can be divided into three simple steps. The first step is imprinting a 3D-printed stamp pattern onto a soft substrate (Figure 2-e), which will later work as a liquid electronics component or a channel. This is a familiar process frequently used when crafting with clay.

The second step is sealing the crafted channel with a transparent tape (Figure 2-f). We introduce a simple and robust DIY method to seal the channel based on a household tape and magnet-based terminals (Section 4.2.3).

The third step is infusing a conductive liquid to the stamped channel (Figure 2-g). We use a transparent sealing medium to visualize the flow of the conductive liquid and demonstrate the inner working process of liquid electronic devices.

Tangible Interactions: The last step is probing the functionality of the fabricated liquid electronic device through tangible interaction. Flowcuits introduces I/O interfaces for fluidics and electronics to associate the manipulation of the conductive liquid resulting 
changes in the electrical properties so that users can intuitively explore the working principle of basic electronic components and circuits (Figure 2-h,i).

Figure 1 depicts a real-life example of Flowcuits workflow to build a liquid LED controller with a resistor stamp integrating I/O interface elements.

\subsection{Flowcuits Stamp Composition}

In order to build a Flowcuits component, three types of elements need to be present in the stamp.

Fluid Elements: These elements design the pattern of reservoirs and channels (tunnels) that can control the flow of conductive liquids. Reservoirs usually hold the liquid, or act as user input interface (e.g., affords pressure input). By connecting the channel with reservoirs, we can guide the flow of the held liquid along the designed channels. To support versatile designs of liquid electronic devices and circuits with the least number of stamps, four types of channels (i.e., line, L-turn, T-junction, cross junction) are proposed as basic flowing elements.

Fluid I/O: These elements implement the fluid ports to infuse the conductive liquid elements. The I/O elements are also essential to drain an excessive amount of the liquids, reduce infusing pressure, and clean out troublesome liquid residues and air bubbles that may degrade the performance of the liquid electrical device.

Electrical I/O: These elements are the electrical probe terminals used to connect LEDs and other electrical wires to observe electrical changes.

\subsection{Flowcuits Tangible-Visual-Electrical Analogy}

Flowcuits follows an easy to understand tangible to visual to the electrical analogy, where tangible inputs are directly reflected in both visual and electrical change as the outputs. In our design, we identified three different analogical patterns that can be used to implement different electrical functions. They are 1) dynamic contact changes, 2) state changes and 3) proportional changes. These three are depicted in Figure 3. Specific examples using individual and combinations of these analogies are presented in Section 5.

Dynamic Contact change: Fluidic circuits can be designed to implement contact or switching mechanisms that respond to tangible user input. As shown in Figure 3-a, a reservoir element with a capillary channel element can be implemented as an open circuit. By applying pressure on the reservoir, the conductive liquid will flow through the channel to close the circuit. The conductive liquid displacement physically visualizes the electrical contact action, which can be simultaneously confirmed with a simple electrical measurement, or with an output element, such as an LED. These changes are dynamic and reversible, and can be used to show the operation of a push button, or a logic gate.

State changes: Similar to contact changes, state change elements can be implemented where the tangible interactions, such as applying pressure makes a state change in the fluidic circuit. In Figure 3-b, a carefully chosen parameters of the channel and reservoir makes the conductive element to transfer from one reservoir to the other This physical process can be easily used in an electrical circuit state change, for instance, to switch the ON/OFF state of an LED. These

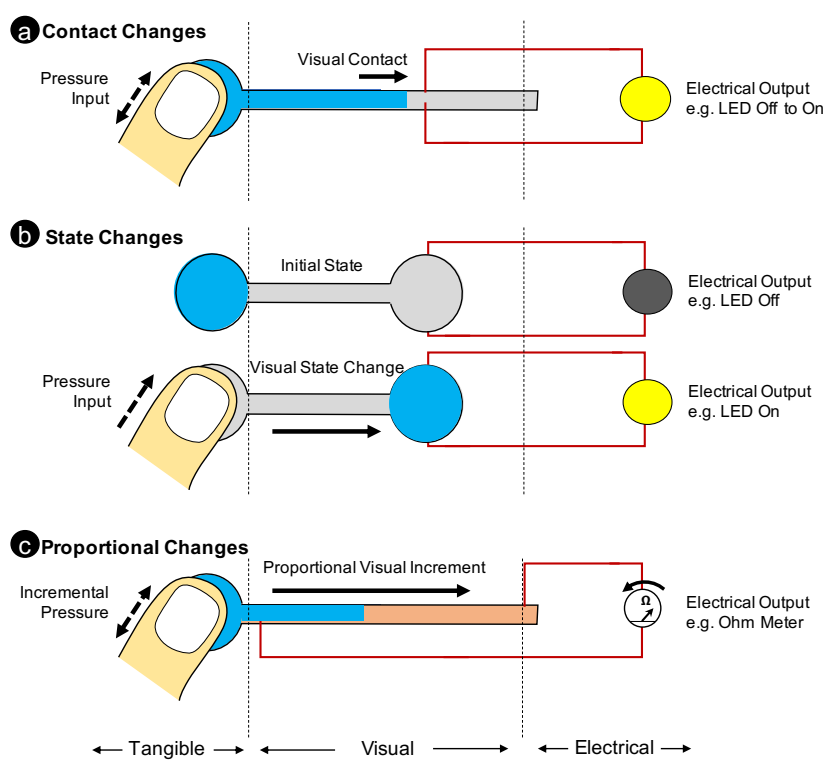

Figure 3: Flowcuits tangible to visual to electrical analogy. a) contact changes, b) state changes and c) proportional changes

changes are stable and hence needs reverse actions to recover to the original state, which can be applied to depict an ON/OFF toggle switch or a flip flop.

Proportional changes: Proportional changes are implemented to depict a gradual response to the input action. Figure 3-c shows a scenario where the length of the conductive liquid in a capillary channel is controlled by the applied pressure at the reservoir. Using two conductive liquids with different electrical properties (e.g., resistivity), this mechanism can be used to change the electrical characteristics of a circuit (e.g., resistance). The input pressure is visually observable with the length of liquid infilling in the channel, and can be measured as an electrical output; for instance, the resistance measured with an Ohm meter will change proportionally to the pressure since the length of the conductive channel changes. This can be used to demonstrate the first law of resistance; namely, the resistance is proportional to the length of the conductor.

\section{DESIGN AND FABRICATION}

\subsection{Flowcuits Stamps 3D Design Toolkit}

To ensure accurate functionality of the Flowcuits components, it is important to consider the geometric effect of each stamp element and properties of conductive liquid. For instance, the flow resistance of a liquid to move along a channel depends on the channel width and depth as well as the viscosity of the liquid.

To help novice users to design and fabricate the 3D printable stamps easily without the knowledge of these conductive fluid parameters, we designed a CAD design software toolkit using Rhinoceros and Grasshopper platform (Figure 4). In our toolkit, users simply drag and drop desired pre-built components of each circuit element (straight, L-turn, T-cross, cross-juction, resistor, 


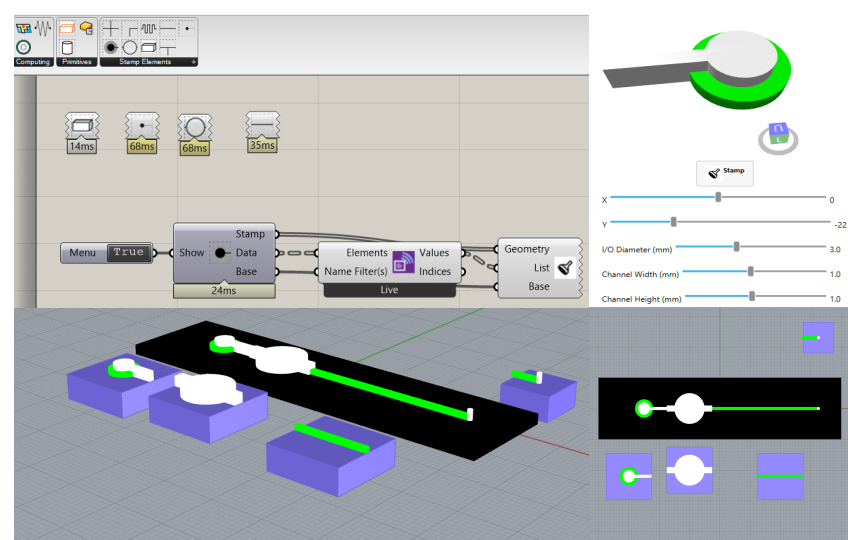

Figure 4: 3D CAD toolkit for the design of custom Flowcuits stamps.

reservoir, inlet/outlet, capacitor) from the ribbon menu and export the design in a STL file to 3D print as a modular stamp consisting of a single element or the assembly of multiple elements. The tool supports six basic circuit elements and their integration circuit. Users can also tune the dimension of each component with a GUI to configure hydraulic and electrical resistance of resistor elements and channel elements, the maximum volume of each reservoirs and the size of $\mathrm{I} / \mathrm{O}$ terminals to match the dimension of an infusion tube adapter.

Our toolkit automatically calculates and informs the required volume and pressure of infusion, hydraulic resistances, and electrical resistance as the user changes the geometric parameters of a rectangular channel and a circular reservoir. The hydraulic resistance is calculated by the equation (from [6]):

$$
R_{\mathrm{H}}=\frac{12 \eta L}{w h^{3}\left(1-\frac{h}{w}\left(\frac{192}{\pi^{5}} \sum_{n=1,5,5}^{\infty} \frac{1}{n^{5}} \tan h\left(\frac{n \pi w}{2 h}\right)\right)\right)}
$$

where $w$ and $h$ are the channel width and height, $L$ is the length of liquid and $\eta$ is the liquid viscosity. Applying Hagen-Poiseuille's Law (similar to Ohm's law $V=R I$ ), a required pressure difference $(\Delta p)$ to drive a liquid flow along the channel at a desired flow rate $(\mathrm{Q})$ is calculated as $\Delta p=Q R_{\mathrm{H}}[40,41]$. To calculate a valve pressure threshold to deform a liquid out of a reservoir to the channel $\left(\Delta p_{\text {valve }}\right)$, the Laplace pressure force (capillary pressure) of the Flowcuits component composed of channel and a reservoir is calculated with Young-Laplace equation: $\Delta p_{v}=\left|p_{\text {reservoir }}-p_{\text {channel }}\right|$ where $p_{\text {reservoir }}=\gamma\left(\frac{2}{D}+\frac{2}{h}\right), p_{\text {channel }}=2 \gamma\left(\frac{1}{w}+\frac{1}{h}\right), \gamma$ is the surface tension of liquid, and $D$ is the diameter of the reservoir.

Finally, our toolkit automatically calculates the electronic resistance of a resistor component from the standard resistance formula: $R=\frac{\rho L}{A}$, where $\rho$ is resistivity, $\mathrm{L}$ is a length of channel and $\mathrm{A}$ is a cross sectional area of the channel. Material properties such as electrical conductivity and viscosity of liquid (e.g., EGaIn liquid metal, $\mathrm{NaOH}, \mathrm{KOH}$, water) and the tested thickness of instant molding substrates (i.e. Blu Tack) are listed in the dropdown menu to select but also customizable by users. Considering the accuracy of a standard desktop 3D printer, the CAD toolkit supports the user to fabricate a fluid channel with the minimum feature size of $500 \mu \mathrm{m}$ at default.

\subsection{Flowcuits Fabrication Process}

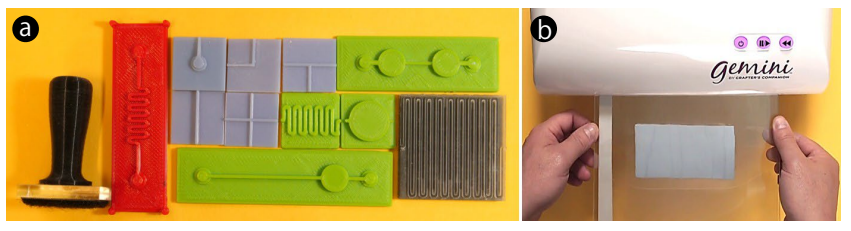

Figure 5: Preparation of Flowcuits stamp sets and molding materials: (a) Prototyped Flowcuits Stamp Elements by a 3D printer and laser cutter. (b) Rolling process to condition the rough surface of Blu Tack.

Flowcuits is built upon rapid prototyped Flowcuits Stamps, an instant reusable molding material (i.e. Blu Tack), a clay rolling machine, a transparent sealing tape, a conductive liquid, infusion toolkit (syringe, teflon tubing, magnetic tube adapter), and probing accessory (i.e. LEDs, conductive wire, battery, multimeter and conductive tape).

4.2.1 Preparation of Flowcuits Stamp and molding Substrate. The first step of Flowcuits is to design Flowcuits stamps by the aforementioned CAD toolkit and prototype them with a 3D printer or a laser cutter. While the CAD toolkit can create a complex liquid electronic system as a single unit of the stamp, Flowcuits also allows a nontechnical user to explore a versatile liquid electronics design by manually stamping prototyped basic modular elements on a molding substrate. As the preparation of the following stamping step, Flowcuits requires a flat and smooth molding substrate to imprint the designed fluid channel accurately. Among many instant reusable molding materials, Flowcuits utilizes a pressure-sensitive adhesive silicone, Blu Tack ${ }^{1}$, as it is not only widely available as an office supply but also seals a liquid with glue-like adhesive surface. Flowcuits utilizes a clay rolling machine (e.g., Gemini machine by Crafters companion ${ }^{2}$ ) to condition an off-the-shelf pressuresensitive adhesive silicone to the smooth and flat molding substrate with a predetermined thickness (e.g., $2 \mathrm{~mm}$ ). To avoid adhesion of the silicone in the rolling machine, the adhesive surface must be rinsed with water and placed between plastic shims that determine the thickness of the substrate after rolling. The adhesiveness will recover by drying the surface. In this paper, we utilized $90 \mathrm{~g}$ of Bostik Blu Tack and prepared flat molding materials with a dimension of $60 \mathrm{~mm} \times 120 \mathrm{~mm} \times 2 \mathrm{~mm}$ through the rolling process.

4.2.2 Repeatable Stamping Process. As the supporting tool to press a small stamp, Flowcuits provides a 3D-printed stamp hand tool which can fix the stamp temporally by utilizing a Velcro tape (Figure 5-a). During the stamping process, the adhesive surface should be covered with a fine-grained powder such as a cornstarch to avoid

\footnotetext{
${ }^{1}$ Bostik Blu Tack (Economy) https://diy.bostik.com/en-UK/products/stationery-craft/ blu-tackr

${ }^{2}$ Gemini machine by Crafters companion https://www.crafterscompanion.com/page/ gemini-machine/
} 


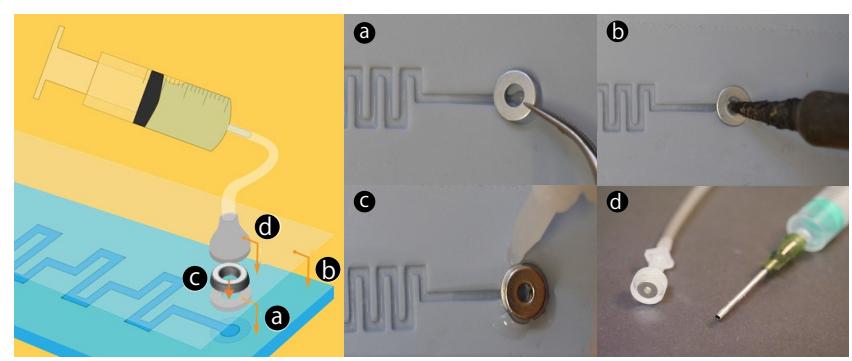

Figure 6: Sealing and Infusion Process: (a) Place a thin gasket/washer at the position of the stamped Fluid I/O element. (b) Seal the entire system with a transparent tape and open the sealed washer hole. (c) Place a magnet ring on top of the washer and seal the edge with a waterproof glue. (d) Create a magnetic infusion tube adapter and attach it to the magnet ring to infuse a conductive liquid with a syringe.

the troublesome adhesion of the molding material within the stamp groove. A 3D-printed stamp must be pressed until the edge of the stamp base reaches the substrate surface from the side view. To avoid over-pressing the stamp into the substrate, the user can optionally supplement supporting pillars at the corners of the stamp in the CAD toolkit, which have the same height as the substrate thickness, as shown in the red 3D-printed stamp in the Figure 5-a. By rolling the surface of the stamped substrate (Figure 5-b), the Flowcuits stamping step allows the user to recycle the failed instant molding material and thus supports the hands-on design process at low cost.

4.2.3 Liquid Sealing and Infusion Process. Figure 6 describes the sealing and infusion process of Flowcuits. At first, a M3 thin gasket/washer $(3.3 \mathrm{~mm} \times 6.0 \mathrm{~mm} \times 0.5 \mathrm{~mm})$ is placed at the position of stamped Fluid I/O elements (Figure 6-a) to support attachment of a magnetic tube adapter later. Next, the entire fluid channel is sealed by a waterproof transparent adhesive tape (e.g., duct tape, wound dressing tape). The sealed Fluid I/O element can be easily opened again by melting the tape with a soldering iron to enable infusion (Figure 6-b). A neodymium magnet ring $(6.0 \mathrm{~mm} \times 2.0 \mathrm{~mm} \times 2.0 \mathrm{~mm}$, $\mathrm{N} 45)$ is placed on top of the washer and fixed to the tape with a waterproof glue around the edge to seal the potential leakage in the following infusion process. A custom magnet ring tube adaptor (Figure 6-c) is built with a laser-cut acrylic magnet ring holder and a 1/16" Female Luer to Hose Barb Adapter (Cole-Parmer). To avoid liquid residues, 1/16" Tygon tubing (Tygon E-3603,Cole-Parmer) is utilized and connected to the adapter to infuse a microlitre level of a conductive liquid with a syringe (Figure 6-d). We observed an adhesive duct tape (e.g., Crystal Clear Gorilla Tape) can seal the channel without leakage up to $50 \mathrm{kPa}$ of the infusion pressure. The maximum pressure force can be enhanced further by sealing the entire device with a DIY soft silicone (e.g., Silicone Addition Transparent 15 Fast). Extra care is required to avoid sealing the fluid I/O ports by filling the silicone up to the surface of the sealed Blu Tack.

4.2.4 Properties of Conductive Liquids. Although there are a variety of conductive liquids, a liquid metal alloy based on Gallium is the

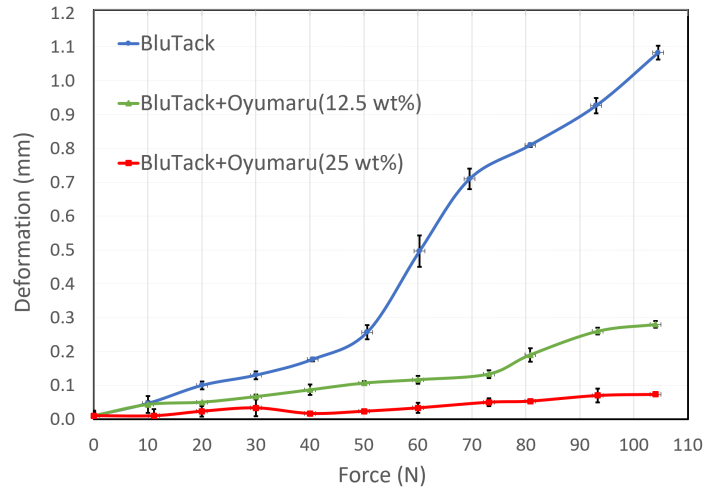

Figure 7: Permanent deformation of Blu Tack and mixed Blu Tack with thermal plastic elastomer (Oyumaru) at room temperature.

most promising material to build liquid electronics system [24, 36 ] due to its high electrical conductivity $(29.4 \mu \Omega \cdot \mathrm{cm})$ [7]. Ionic solutions (e.g., saline water, alkaline solution) are also applicable to design conductive fluidic channels although they have relatively low order of conductivity $[12,40]$. Combining conductivity with surface tension, users can build a momentary switch based on liquid deformation (see Figure 8). While a liquid metal has shiny metallic color, most of the ionic liquids are transparent and difficult to trace. By adding color dye such as food color ink or $\mathrm{pH}$ indicator, users can visualize the liquid flow to probe the inner working of the fabricated liquid electronic system.

4.2.5 Evaluation of Molding Material Robustness to Pressing Force. While a Blu Tack is useful to rapid prototype a fluid channel with Flowcuits Stamps, there is a trade-off to gain permanent deformation under a significant pressure during interaction (e.g., strong finger press). We built a custom measurement setup consisting of a $12 \mathrm{~mm}$ diameter resistive force sensor (Interlink 402 model) and a precision vice to control the force. We measured the deformation with finger-sized samples $(15 \mathrm{~mm} \times 15 \mathrm{~mm} \times 2 \mathrm{~mm})$. Experiment was conducted three times at a room temperature. Figure 7 shows the average values of the measured deformation.

Blu Tack yielded above $50 \mathrm{~N}$ force and gained $1.07 \pm 0.02 \mathrm{~mm}$ deformation over the force of $100 \mathrm{~N}$. As the average force of a pad press was reported around $45 \mathrm{~N}$ with an index finger [1], a Blu Tack can likely deform the channel around $0.2 \mathrm{~mm}$ depth for a regular pressing interaction. To enhance the robustness of Blu Tack, we explored a hybrid material combining a Blu Tack with a thermoplastic elastomer, called Oyumaru. Oyumaru is another popular DIY reusable molding material that becomes moldable by heating $\left(70-100^{\circ} \mathrm{C}\right)$ and can recover the rigid elasticity after cooling down ${ }^{3}$. However, because the surface of thermoplastic elastomers are smooth/slick like Teflon, it is difficult to seal the stamped channel with adhesive tapes. Like a clay, kneading a Blu Tack with a small portion of heated Oyumaru created a hybrid molding material that is adhesive and more elastic than a Blu Tack.

\footnotetext{
${ }^{3}$ Oyumaru Reusable Molding Material https://thebluebottletree.com/oyumarureusable-molding-material/
} 

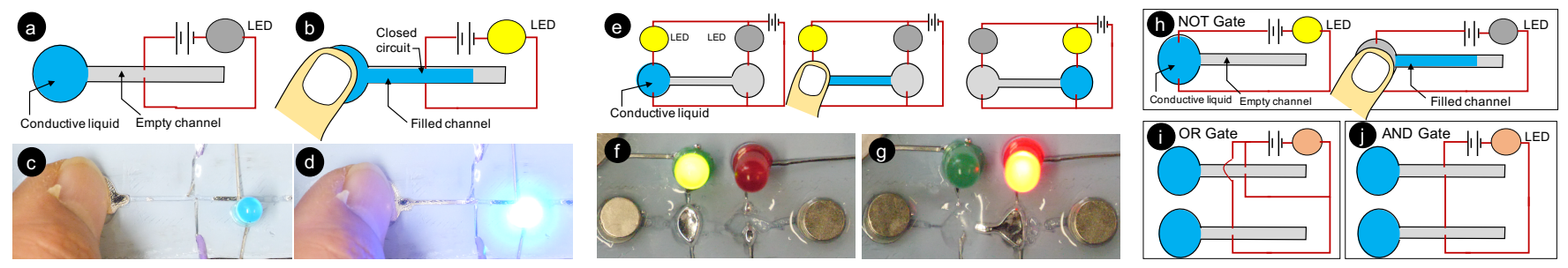

Figure 8: An interactive push-button style switch (a, b, c, d); a) stable or OFF state where the conductive liquid is pooled inside the reservoir; b) application of pressure force the conductive liquid to channel making the circuit to be closed creating ON state; $c, d$ ) implementation of the push-button. An interactive toggle switch (e, f, g); e) block diagram of the interactive toggle switch showing two reservoirs and two LEDs; $f, g$ ) real implementation of the left toggled state; b) real implementation of the right toggled state. Digital gates $(h, i, j) ; h)$ NOT Gate where circuit is normally closed at the reservoir and opens when pressed by displacing liquids to the channel; i) OR gate where two channels are connected as parallel switches; j) AND gate where two switches are connected in series.

Figure 7 shows the significant improvement of the hybrid material in the yield strength. For instance, 12.5 (wt\%) ratio sample gained $0.10 \pm 0.01 \mathrm{~mm}$ deformation at $50 \mathrm{~N}$ and $0.27 \pm 0.01 \mathrm{~mm}$ above $100 \mathrm{~N}$ while $25(w t \%)$ ratio sample showed $0.01 \pm 0.01 \mathrm{~mm}$ at $50 \mathrm{~N}$ and $0.06 \pm 0.01 \mathrm{~mm}$ above $100 \mathrm{~N}$. However, as the hybrid Blu Tack loses the adhesiveness by containing more thermoplastic elastomers, the user must consider a trade-off of the channel sealing capability and the robustness to interaction based on the application. For general use, we recommend 12.5 ( $w t \%)$ as a good compromise.

\section{APPLICATION SCENARIOS}

In this section, we describe how Flowcuits can be used to demonstrate the operation of fundamental electronic components. Later we show how these basic components can be used to construct complex circuits.

\subsection{Interactive Switches and Digital Gates}

Using Flowcuits toolkit, we created two basic types of interactive switches. One is a push-button style reversible switch and the other is a toggle style persistent switch. We selected these two types of switches since they can be used to create digital electronic elements, as outlined at the end of the section.

5.1.1 Push-Button Switch. Push-button style switches help us to make a transient element, which can be analogous to a simplified not-gate. To create a interactive ON-OFF push button style switch, we use a stable reservoir component and a connected capillary channel. As shown in Figure 8-a, a circuit is created with two terminals at the either side of the channel, making an open circuit across the channel. Once the reservoir membrane is pressed as shown in Figure 8-b, conductive liquid displaces into the channel closing the circuit, turning the LED ON. Figure 8-(c, d) show the $\mathrm{ON}$ and OFF states of the real implementation. When users release the pressure, conductive liquid reverts back to the reservoir due to its surface tension based Laplace pressure force and air pressure, causing the circuit to revert back to OFF state. Therefore, this switch acts like a push-button style reversible component.

5.1.2 Toggle Switch. Toggle switches are analogous to components with simple memory, where actions are persistent. To create a simple toggle switch with Flowcuits, we use two stable reservoirs connected with a capillary channel as shown in Figure 8-e. Two electric paths are created with open-circuits across each reservoir to demonstrate each condition. When one of the reservoirs membrane is pressed, the liquid starts to flow through the channel to the other reservoir as shown in the progression of images from left to right in Figure 8-e. This makes first circuit to turn-OFF and second to turn$\mathrm{ON}$. Since the surface tension is stable in the reservoir, this state is stable and would persist even if the finger is removed. Figure 8-f and $g$ show the each state of the real implementation.

5.1.3 Digital Gates. Flowcuits switches can be used to create interactive gates to demonstrate basic digital logic. In Figure 8-(h, i, j), we demonstrate how logic gates (NOT, OR and AND) can be made with a reservoirs and capillary channels. The set of connectives \{NOT, AND $\}$ is a functionally complete Boolean operators, meaning any other gate can be made using combination of these.

NOT Gate: Figure 8 -h shows the implementation of a NOT gate with Flowcuits. The circuit is completed through the reservoir (as oppose to the channel) so that when an input (a finger press) is not present (input 0 ), the output LED is ON indicating a logic output 1. When the pressure is applied (input 1), conductive liquid displaces to the channel making the reservoir empty. This breaks the circuit and turn the LED OFF, creating a logic output 0 .

OR Gate: Figure 8-i shows the implementation of an OR gate with Flowcuits connecting two push-button style switches in parallel. If either of the reservoirs are pressed (logic input 01,10 or 11), the output LED is ON indicating a logic output 1 . LED is OFF only when the input is 00 .

AND Gate: As shown in Figure 8-j, an AND gate can be implemented connecting two push-button style switches in series. The output LED is ON (logic output 1) only if both reservoirs are pressed, i.e. input is 11 .

\subsection{Analog Electronic Components}

We created three analog electronic components, i.e., a variable resistor, a pressure sensor and a variable capacitor, with Flowcuits as application demonstrators. 


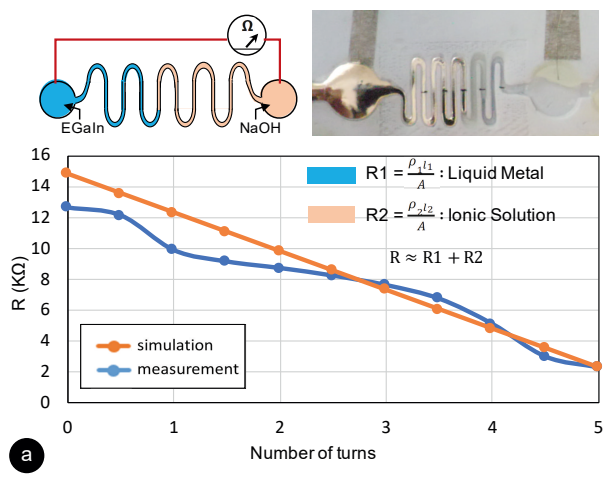

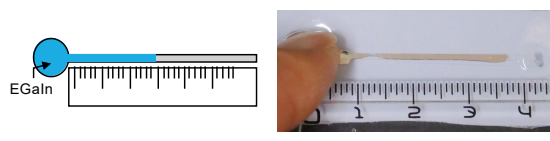

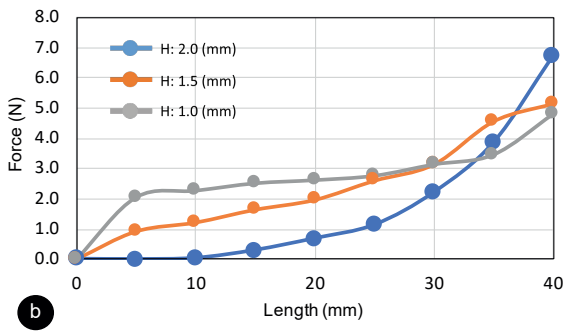

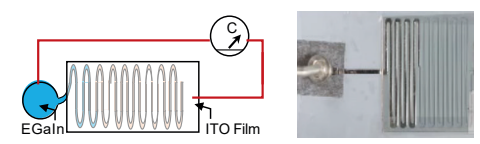

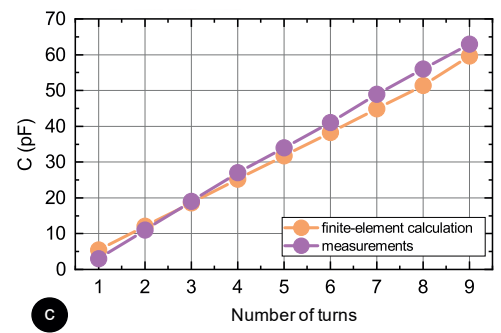

Figure 9: Analog Electronic Components. a) A variable resistor design, implementation and measurement results; b) A pressure sensor design, implementation and measurement results; c) A variable capacitor design, implementation and measurement results.

5.2.1 Variable Resistor and Pressure Sensor. Resistors are probably the most frequently used components in analog circuitry. When demonstrating the principles of electrical resistance (e.g., the relationship between an electrical resistance and the length, area and resistivity of a conductive material), a variable resistor or a variac can be extremely useful.

To implement a variable resistor with Flowcuits, we used two conductive liquids with different resistivities, 1) $\mathrm{NaOH}$ electrolyte as high resistance solution $(7.94 \mathrm{k} \Omega \cdot \mathrm{cm})$ and 2$)$ a liquid metal eutectic (EGaIn, $29.4 \mu \Omega \cdot \mathrm{cm}$ ) as low resistance solution. This Flowcuits prototype was created with two reservoirs connected with a serpentine patterned channel as shown in Figure 9-a. A serpentine pattern was used to increase the effective length. Then conductive metal and $\mathrm{NaOH}$ solutions were infused to two reservoirs from either side. When measured across two reservoirs, this creates a series resistance with the low resistive liquid metal and high resistive $\mathrm{NaOH}$. By controlling the length of the each fluid component, we can control the resistance. Since the top shield of Flowcuits is transparent, observers can see the change of length (as shown in the real image of Figure 9-a).

To demonstrate the operation of the Flowcuits variable resistor, we measured the relationship between the resistance value and the volume ratio of the liquid metal to $\mathrm{NaOH}$ solution at eleven evenly divided points at the center of the resistor pattern. We placed two silver wires at each end point of the resistor and probed them with an LCR meter (MS5308). This Flowcuits used a $1.0 \mathrm{~mm}^{2}$ square channel with two reservoirs $(6.0 \mathrm{~mm}(\phi) \times 2.0 \mathrm{~mm}(H))$. We compared the measurement with a calculated theoretical value from a parallel resistor model. For the experiment we used a manual syringe infusion to accurately control the length. As shown in Figure 9-a graph, we observed the proportional decrease of the resistance with $2.0 \mathrm{k} \Omega$ maximum deviation from the model. Furthermore, since $\mathrm{NaOH}$ electrolytes helps to remove oxide-skins of EGaIn, which helps to increase the fluid resistance because of gel-like high viscosity and surface energy [59]; we could retract back the liquid metal layer back to the reservoir to make the effect reversible.

Likewise, a pressure sensor can be made by combining one reservoir with a straight capillary channel, as shown in Figure 9-b.
By applying pressure on the reservoir, conductive liquid is displaced into the channel. The length of the liquid metal in the channel is used as a measure of pressure in the reservoir. To understand the relationship between the exerted pressure and the displacement length of liquid metal, we measured the force and the displacement length for three different square channel sizes $(1.0 \mathrm{~mm} \times 1.0 \mathrm{~mm}, 1.0 \mathrm{~mm} \times 1.5 \mathrm{~mm}$, and $1.0 \mathrm{~mm} \times 2.0 \mathrm{~mm})$. Our goal was to identify the minimum viable channel size, since the smaller the channel, higher the irregularities of the channel shape, causing inconsistencies in the flow. We measured the force using a $12 \mathrm{~mm}$ diameter resistive force sensor (Interlink 402 model) underneath the reservoir. Graph shown in Figure 9-b shows the relationship between the displacement length and the force. Out of the three sizes, $1.0 \mathrm{~mm} \times 2.0 \mathrm{~mm}$ shows the best performance.

5.2.2 Variable Capacitor. Capacitors are another commonly used components in analog circuitry. Similar to the variable resistor, a Flowcuits can be used to create a variable capacitor to show how capacitance of a two-plate capacitor change with the area.

We used an elongated serpentine pattern channel with a reservoir and an infusion inlet to create the variable capacitor. Figure 9-c shows the block diagram and the real image of the variable capacitor. The liquid metal in the capillary channel creates one plate of the capacitor. For the other plate, we used an ITO film $(40 \mathrm{~mm} \times 40 \mathrm{~mm})$ over the channel. This provides the conductive plate without occluding the view of the liquid infusion. The channel is square shaped $\left(1.0 \mathrm{~mm}^{2}\right)$ with $1.0 \mathrm{~mm}$ pitch on a Blu Tack layer $(40 \mathrm{~mm} \times 40 \mathrm{~mm})$.

To verify the operation, we used silver wires connected to each terminal of electrodes and measured the capacitance with an LCR meter (MS5308). The graph shown in Figure 9-c compares the measured values against a set of calculated values using a finite-element analysis using COMSOL Multiphysics. Comparison shows a linear increment of capacitance in agreement with the theoretical model.

\section{DISCUSSION AND LIMITATION}

\subsection{Form factor}

Creating a complex and large Flowcuits circuit requires preparing a large substrate area. An embossing machine maybe used to prepare 
A4 size substrate for the circuit. We recommend a substrate thickness of $3.0 \mathrm{~mm}$ to allow embossing manually using the 3D-printed stamps. Manipulating the liquid metal by pressing the substrate deteriorated with thinner substrate. The width and depth of the channels in the circuits were limited by the feature sizes of the stamps. Sub-millimeter scale wide and deep features can be made using a desktop 3D printer. However, we found that sub-millimeter size channels were not reliable as the connections often broke when pressing the substrate to manipulate the liquid metal. We recommend a minimum of $1.0 \mathrm{~mm}$ wide and $1.0 \mathrm{~mm}$ deep channel for reliably pressing the substrate to push and flow the liquid metal without breaking the circuit. We also found that large channels were difficult to use as the liquid metal could flow backwards due to its high surface tension. Large force was required to move the liquid metal which could permanently deform the shape of the channel. We recommend a maximum of $2.0 \mathrm{~mm}$ wide or $2.0 \mathrm{~mm}$ deep channel, and preferably with depth more than the width for more reliable and comfortable interaction.

\subsection{Safety}

Flowcuits is built with off-the-shelf household materials and standard chemicals that can be safely handled. The instant molding substrates (i.e. Blu Tack, Oyumaru) are used as standard office supplies or DIY modeling clay for hobbyists. Flowcuits utilizes a safe liquid metal that is an eutectic alloy of galium and indium (EGaIn) [7]. As the CRC Handbook of Chemistry and Physics [25] reports both galium and indium with the low orders of toxicity, the eutectic mixture of these metals is used as the alternative of mercury in oral thermometers and even used as a dental filling [4].

Although a Sodium Hydroxide solution $(\mathrm{NaOH}$, known as caustic soda or lye) is used in a standard household cleaning bleach, it is corrosive and thus must be carefully treated to avoid the excessive exposure which may burn skin or eyes. We recommend the user to dilute the $\mathrm{NaOH}$ solution to the levels of concentration that are not harmful for humans (e.g., 0.05 M or less) [52]. Extreme care should be taken to prepare the diluted solution from raw $\mathrm{NaOH}$ solutions which are highly concentrated. The user may wear gloves, goggles and protective clothing to avoid accidental contact with skin and eyes. Upon skin contact, the area should be washed thoroughly under running water. Caustic soda solution can be neutralized with weak acid like vinegar before washing. Upon eye contact, ingestion or inhalation medical advice should be sought along with washing. Because Flowcuits utilizes a few microliters of $\mathrm{NaOH}$ solution in the sealed channels, it is safe to interact with Flowcuits devices with bare hands. However, the user should be aware of the risk of leakage, in particular, if the reservoir is pressed harder than the maximum sealing pressure of $50 \mathrm{kPa}$.

\subsection{Material Reusability}

Dried up liquid metals of Gallium are rapidly oxidized in air and stick to tubing and substrate, leaving thin black residues over the surfaces [7]. $\mathrm{NaOH}$ solution easily removes the residual oxidized layers of liquid metal as small droplets which can be reused. Due to the high surface tension, these small droplets tend to merge into a single large droplet which is easier to collect with a syringe. As $\mathrm{NaOH}$ solution is a common drain cleaner, it can be flushed down the drain after dilution. The sealing tape cannot be reused due to its strong adhesion to Blu Tack. The substrate can be reused but might need washing with $\mathrm{NaOH}$ solution to remove any residual liquid-metal and then $\mathrm{NaOH}$ can be washed with water afterwards. The circuit can be cut with a knife and most of the base substrate can be reused discarding any dirty substrate. Flowcuits allows many standard electronic components like LEDs to be reused as they are not soldered.

\subsection{Applications in Playful Learning and Future Works}

Flowcuits approach leverage on familiar knowledge in malleable molding substrates such as clay to fabricate fundamental electronics components. We believe this is a significant technical enabler for playful learning through iterative exploration. Particularly, the DIY aspects and the tangible-visual-electrical analogy will combine familiar knowledge with unfamiliar and novel to strengthen the learning process of fundamental electrical principles. Furthermore, to the best of our knowledge, no such tangible toolkit exists to broaden the understanding of the inner working of fundamental electronic elements. However, we have not evaluated this through a user study and it remains to be explored in future work. This paper aims to focus on the underlying crafting approach as the enabling technology, the analogy, and the demonstration applications.

In our future work, we plan to further investigate the design space of DIY reconfigurable liquid electronics. Although reconfigurable electronics have been extensively studied, in particular, for the application of radio-frequency $(\mathrm{RF})$ tunner [23, 45], the requirement of complex fluid channel fabrication process (e.g., soft lithography) and special facilities (e.g., plasma cleaner) have hindered the user from exploring novel opportunities for reconfigurable electronics at home. As we demonstrated in Application Scenarios, Flowcuits enables the simple DIY fabrication of reconfigurable electronic components (e.g., pressure-sensitive switch and logic gate, variable resistor, variable capacitor). By integrating the hand-made reconfigurable electronic components into conventional analogue circuits (e.g., RC filter, op-amp circuits), the user could easily design and test reconfigurable electronic systems (e.g., radio tunner, PWM signal generator) by exploring the visual response of conductive liquids to interaction. Extension of more electrical I/O terminals to the liquid channel would expand the more possibilities for playful physical computing by sensing the continuous deformation and movement of infilled conductive liquids with micro-controllers (e.g., Arduino). For instance, because Blu Tack is adhesive soft material designed to attach light weight objects to a smooth surface, the user can bind liquid electronic components (e.g., variable resistor or capacitor) to various daily objects (e.g., paper, plastic sheet, pen, cup, dish) and environments (e.g., wall, door, white board) to implement touch, pressure, and bend sensing capabilities for tangible interaction.

As the auxiliary step to bind multiple I/O terminals to the liquid channel without the risk of liquid leakages, we recommend the use of a DIY silicone elastomer (e.g., Silicone Addition Transparent 15 Fast) to seal the entire liquid electronic component after binding cables to the terminals. 


\section{CONCLUSION}

In this paper, we presented Flowcuits, a DIY fabrication method to prototype tangible, interactive and functional electronic components by manipulating liquid metal mechanisms. Flowcuits uses imprinting conductive fluidic channels on malleable substrates to create electrical circuits and interactive elements. Utilizing galliumindium (Ga-In) liquid metal as the conductive element, we demonstrated our approach can create interactive and customizable electronic components such as switches, variable resistors, variable capacitors, logic gates and pressure sensors. Flowcuits follows a simple tangible - visual - electrical relationship which can be used as an analogy to explore unknown fundamentals through known concepts. Our technical evaluation confirms the functional validity of the proposed concept. We believe that Flowcuits will encourage designers, makers and researchers to create tangible interactive devices for innovative use-cases in learning, art and other related fields.

\section{ACKNOWLEDGMENTS}

This work was funded by EPSRC grant EP/N013948/1 and the Rutherford fellowship scheme to support the visit of Dr. Anusha Withana to Sussex University. Further funding was provided by Australian Research Council Discovery Early Career Award (DECRA) - DE200100479. Dr. Withana is the recipient of a DECRA funded by the Australian Government.

\section{REFERENCES}

[1] Angela DiDomenico Astin. 1999. Finger force capability: measurement and prediction using anthropometric and myoelectric measures. Ph.D. Dissertation. Virginia Tech.

[2] Christoph Bader, William G. Patrick, Dominik Kolb, Stephanie G. Hays, Steven Keating, Sunanda Sharma, Daniel Dikovsky, Boris Belocon, James C. Weaver, Pamela A. Silver, and Neri Oxman. 2016. Grown, Printed, and Biologically Augmented: An Additively Manufactured Microfluidic Wearable, Functionally Templated for Synthetic Microbes. 3D Printing and Additive Manufacturing 3, 2 (Jul. 2016), 79-89. https://doi.org/10.1089/3dp.2016.0027

[3] Ayah Bdeir and Ted Ullrich. 2011. Electronics as material: Littlebits. In Proceedings of the fifth international conference on Tangible, embedded, and embodied interaction - TEI '11. ACM Press, New York, New York, USA, 341. https: //doi.org/10.1145/1935701.1935781

[4] Lee C Cadwallader. 2003. Gallium safety in the laboratory. Technical Report Idaho National Laboratory (INL).

[5] Joshua Chan, Tarun Pondicherry, and Paulo Blikstein. 2013. LightUp: An Augmented, Learning Platform for Electronics. In Proceedings of the 12th International Conference on Interaction Design and Children (New York, New York, USA) (IDC '13). ACM, New York, NY, USA, 491-494. https://doi.org/10.1145/2485760.2485812

[6] RJ Cornish. 1928. Flow in a pipe of rectangular cross-section. Proceedings of the Royal Society of London. Series A, Containing Papers of a Mathematical and Physical Character 120, 786 (1928), 691-700.

[7] Michael D. Dickey, Ryan C. Chiechi, Ryan J. Larsen, Emily A. Weiss, David A. Weitz, and George M. Whitesides. 2008. Eutectic Gallium-Indium (EGaIn): A Liquid Metal Alloy for the Formation of Stable Structures in Microchannels at Room Temperature. Advanced Functional Materials 18, 7 (Apr. 2008), 1097-1104. https://doi.org/10.1002/adfm.200701216

[8] P. Dourish. 2001. Where the Action is: The Foundations of Embodied Interaction. MIT Press, Cambridge, MA, USA.

[9] Elenco. 2020. Snap Circuits Jr. - Elenco Electronics. https://www.elenco.com/ product/snap-circuits-jr-100-experiments/. Last Accessed: 2020-05-01.

[10] A. Fassler and C. Majidi. 2013. Soft-matter capacitors and inductors for hyperelastic strain sensing and stretchable electronics. Smart Materials and Structures 22, 5 (may 2013), 055023. https://doi.org/10.1088/0964-1726/22/5/055023

[11] M. Garrad, G. Soter, A. T. Conn, H. Hauser, and J. Rossiter. 2019. A soft matter computer for soft robots. Science Robotics 4, 33 (aug 2019), eaaw6060. https: //doi.org/10.1126/scirobotics.aaw6060

[12] Leah A. Godwin, Kennon S. Deal, Lauren D. Hoepfner, Louis A. Jackson, and Christopher J. Easley. 2013. Measurement of microchannel fluidic resistance with a standard voltage meter. Analytica Chimica Acta 758 (Jan. 2013), 101-107. https://doi.org/10.1016/j.aca.2012.10.043
[13] Saul Greenberg and Chester Fitchett. 2001. Phidgets: Easy Development of Physical Interfaces Through Physical Widgets. In Proceedings of the 14th Annual ACM Symposium on User Interface Software and Technology (Orlando, Florida) (UIST '01). ACM, New York, NY, USA, 209-218. https://doi.org/10.1145/502348. 502388

[14] Richard Gunstone and Pamela Mulhall. 2009. Physics Teachers' Perceptions of the Difficulty of Teaching Electricity. Research in Science Education 39, 4 (Aug. 2009), 515-538. https://doi.org/10.1007/s11165-008-9092-y

[15] Teng Han, Fraser Anderson, Pourang Irani, and Tovi Grossman. 2018. HydroRing. In The 31st Annual ACM Symposium on User Interface Software and TechnologyUIST '18. ACM Press, New York, New York, USA, 913-925. https://doi.org/10. $1145 / 3242587.3242667$

[16] C. Hoyles and R. Noss. 1999. Playing with (and without) words. In Proceedings of the seventh European Logo Conference (Eurologo'99). Sofia, Bulgaria, 18-29.

[17] Myoung Huh, Dong-Joon Won, Joong Gil Kim, and Joonwon Kim. 2017. Simple and robust resistive dual-axis accelerometer using a liquid metal droplet. Micro and Nano Systems Letters 5, 1 (Jan. 2017), 5. https://doi.org/10.1186/s40486-0160038-2

[18] Yuki Inoue, Yuichi Itoh, and Takao Onoye. 2018. TuVe. In SIGGRAPH Asia 2018 Emerging Technologies on - SA '18. ACM Press, New York, New York, USA, 1-2. https://doi.org/10.1145/3275476.3275487

[19] Seung Hee Jeong, Anton Hagman, Klas Hjort, Magnus Jobs, Johan Sundqvist, and Zhigang Wu. 2012. Liquid alloy printing of microfluidic stretchable electronics. Lab Chip 12 (2012), 4657-4664. Issue 22. https://doi.org/10.1039/C2LC40628D

[20] Samuel Johnson and AnnMarie P. Thomas. 2010. Squishy Circuits: A Tangible Medium for Electronics Education. In CHI '10 Extended Abstracts on Human Factors in Computing Systems (Atlanta, Georgia, USA) (CHI EA '10). ACM, New York, NY, USA, 4099-4104. https://doi.org/10.1145/1753846.1754109

[21] Yoshihiro Kawahara, Steve Hodges, Benjamin S. Cook, Cheng Zhang, and Gregory D. Abowd. 2013. Instant inkjet circuits: lab-based inkjet printing to support rapid prototyping of UbiComp devices. In Proceedings of the 2013 ACM international joint conference on Pervasive and ubiquitous computing - UbiComp '13. ACM Press, New York, New York, USA, 363. https://doi.org/10.1145/2493432.2493486

[22] Nicholas Kellaris, Vidyacharan Gopaluni Venkata, Garrett M. Smith, Shane K. Mitchell, and Christoph Keplinger. 2018. Peano-HASEL actuators: Musclemimetic, electrohydraulic transducers that linearly contract on activation. Science Robotics 3, 14 (Jan. 2018), eaar3276. https://doi.org/10.1126/scirobotics.aar3276

[23] M. Kelley, C. Koo, H. Mcquilken, B. Lawrence, S. Li, A. Han, and G. Huff. 2013. Frequency reconfigurable patch antenna using liquid metal as switching mechanism. Electronics Letters 49, 22 (Oct. 2013), 1370-1371. https: //doi.org/10.1049/el.2013.2930

[24] Min-gu Kim, Hommood Alrowais, Spyridon Pavlidis, and Oliver Brand. 2017. Size-Scalable and High-Density Liquid-Metal-Based Soft Electronic Passive Components and Circuits Using Soft Lithography. Advanced Functional Materials 27, 3 (Jan. 2017), 1604466. https://doi.org/10.1002/adfm.201604466

[25] David R Lide. 2007. CRC Handbook of Chemistry and Physics. (2007)

[26] Emily Lovell and Leah Buechley. 2010. An e-Sewing Tutorial for DIY Learning. In Proceedings of the 9th International Conference on Interaction Design and Children (Barcelona, Spain) (IDC '10). ACM, New York, NY, USA, 230-233. https://doi. org $/ 10.1145 / 1810543.1810578$

[27] Qiuyu Lu, Chengpeng Mao, Liyuan Wang, and Haipeng Mi. 2016. LIME. In Proceedings of the 29th Annual Symposium on User Interface Software and Technology - UIST '16. ACM Press, New York, New York, USA, 449-452. https: //doi.org/10.1145/2984511.2984562

[28] Oiuyu Lu, Jifei Ou, João Wilbert, André Haben, Haipeng Mi, and Hiroshi Ishii. 2019. milliMorph - Fluid-Driven Thin Film Shape-Change Materials for Interaction Design. In Proceedings of the 32nd Annual ACM Symposium on User Interface Software and Technology. ACM, New York, NY, USA, 663-672. https://doi.org/10.1145/3332165.3347956

[29] Tong Lu, Lauren Finkenauer, James Wissman, and Carmel Majidi. 2014. Rapid Prototyping for Soft-Matter Electronics. Advanced Functional Materials 24, 22 (2014), 3351-3356. https://doi.org/10.1002/adfm.201303732 arXiv:https://onlinelibrary.wiley.com/doi/pdf/10.1002/adfm.201303732

[30] Robert MacCurdy, Robert Katzschmann, Youbin Kim, and Daniela Rus. 2016. Printable hydraulics: A method for fabricating robots by 3D co-printing solids and liquids. In 2016 IEEE International Conference on Robotics and Automation (ICRA), Vol. 2016-June. IEEE, 3878-3885. https://doi.org/10.1109/ICRA.2016.7487576

[31] David A. Mellis, Leah Buechley, Mitchel Resnick, and Björn Hartmann. 2016. Engaging Amateurs in the Design, Fabrication, and Assembly of Electronic Devices. In Proceedings of the 2016 ACM Conference on Designing Interactive Systems (Brisbane, QLD, Australia) (DIS '16). ACM, New York, NY, USA, 1270-1281. https://doi.org/10.1145/2901790.2901833

[32] David A. Mellis, Sam Jacoby, Leah Buechley, Hannah Perner-Wilson, and Jie Oi. 2013. Microcontrollers As Material: Crafting Circuits with Paper, Conductive Ink, Electronic Components, and an "Untoolkit". In Proceedings of the 7th International Conference on Tangible, Embedded and Embodied Interaction (Barcelona, Spain) (TEI '13). ACM, New York, NY, USA, 83-90. https://doi.org/10.1145/2460625. 
2460638

[33] R. Hunter Montgomery and Bryant C. Hollins. 2016. Fabrication method for paper microfluidics utilizing 3D printing and PDMS stamps. Proceedings - 32nd Southern Biomedical Engineering Conference, SBEC 2016 (2016), 64-65. https: //doi.org/10.1109/SBEC.2016.70

[34] Hila Mor, Tianyu Yu, Ken Nakagaki, Benjamin Harvey Miller, Yichen Jia, and Hiroshi Ishii. 2020. Venous Materials: Towards Interactive Fluidic Mechanisms. In Proceedings of the 2020 CHI Conference on Human Factors in Computing Systems. ACM, New York, NY, USA, 1-14. https://doi.org/10.1145/3313831.3376129

[35] Alex J.L. Morgan, Lorena Hidalgo San Jose, William D. Jamieson, Jennifer M Wymant, Bing Song, Phil Stephens, David A. Barrow, and Oliver K. Castell. 2016. Simple and versatile 3D printed microfluidics using fused filament fabrication. PLoS ONE 11, 4 (2016), 1-17. https://doi.org/10.1371/journal.pone.0152023

[36] Steven Nagels, Raf Ramakers, Kris Luyten, and Wim Deferme. 2018. Silicone Devices. In Proceedings of the 2018 CHI Conference on Human Factors in Computing Systems - CHI '18. ACM Press, New York, New York, USA, 1-13. https://doi.org/ $10.1145 / 3173574.3173762$

[37] Koya Narumi, Xinyang Shi, Steve Hodges, Yoshihiro Kawahara, Shinya Shimizu, and Tohru Asami. 2015. Circuit Eraser: A Tool for Iterative Design with Conductive Ink. In Proceedings of the 33rd Annual ACM Conference Extended Abstracts on Human Factors in Computing Systems - CHI EA '15. ACM Press, New York, New York, USA, 2307-2312. https://doi.org/10.1145/2702613.2732876

[38] Ryuma Niiyama, Lining Yao, and Hiroshi Ishii. 2013. Weight and volume changing device with liquid metal transfer. In Proceedings of the 8th International Conference on Tangible, Embedded and Embodied Interaction - TEI '14, Vol. C. ACM Press, New York, New York, USA, 49-52. https://doi.org/10.1145/2540930.2540953

[39] Thomas O'Brien. 1993. Teaching Fundamental Aspects of Science Toys. School Science and Mathematics 93, 4 (Apr. 1993), 203-207. https://doi.org/10.1111/j.19498594.1993.tb12225.x

[40] Kwang W Oh, Kangsun Lee, Byungwook Ahn, and Edward P Furlani. 2012. Design of pressure-driven microfluidic networks using electric circuit analogy. Lab on $a$ Chip 12, 3 (2012), 515-545.

[41] Ayokunle Olanrewaju, Maïwenn Beaugrand, Mohamed Yafia, and David Juncker 2018. Capillary microfluidics in microchannels: from microfluidic networks to capillaric circuits. Lab on a Chip 18, 16 (2018), 2323-2347. https://doi.org/10. 1039/C8LC00458G

[42] Yong-Lae Park, Carmel Majidi, Rebecca Kramer, Phillipe Bérard, and Robert J. Wood. 2010. Hyperelastic pressure sensing with a liquid-embedded elastomer. fournal of Micromechanics and Microengineering 20, 12 (dec 2010), 125029. https: //doi.org/10.1088/0960-1317/20/12/125029

[43] Sara Price and Yvonne Rogers. 2004. Let's Get Physical: The Learning Benefits of Interacting in Digitally Augmented Physical Spaces. Comput. Educ. 43, 1-2 (2004), 137-151. https://doi.org/10.1016/j.compedu.2003.12.009

[44] S. Price, Y. Rogers, M. Scaife, D. Stanton, and H. Neale. 2003. Using 'tangibles' to promote novel forms of playful learning. Interacting with Computers 15, 2 (Apr. 2003), 169-185. https://doi.org/10.1016/S0953-5438(03)00006-7

[45] Mohammad Rashed Khan, Gerard J. Hayes, Ju-Hee So, Gianluca Lazzi, and Michael D. Dickey. 2011. A frequency shifting liquid metal antenna with pressure responsiveness. Applied Physics Letters 99, 1 (jul 2011), 013501. https: //doi.org/10.1063/1.3603961

[46] M. Resnick, F. Martin, R. Sargent, and B. Silverman. 1996. Programmable Bricks: Toys to Think with. IBM Syst. 7. 35, 3-4 (1996), 443-452. https://doi.org/10.1147/ sj.353.0443

[47] Yvonne Rogers, Jeni Paay, Margot Brereton, Kate L. Vaisutis, Gary Marsden, and Frank Vetere. 2014. Never Too Old: Engaging Retired People Inventing the Future with MaKey MaKey. In Proceedings of the SIGCHI Conference on Human Factors in Computing Systems (Toronto, Ontario, Canada) (CHI '14). ACM, New York, NY, USA, 3913-3922. https://doi.org/10.1145/2556288.2557184

[48] Deepak Ranjan Sahoo, Timothy Neate, Yutaka Tokuda, Jennifer Pearson, Simon Robinson, Sriram Subramanian, and Matt Jones. 2018. Tangible Drops. In Proceedings of the 2018 CHI Conference on Human Factors in Computing Systems CHI '18. ACM Press, New York, New York, USA, 1-14. https://doi.org/10.1145/ 3173574.3173751

[49] Valkyrie Savage, Ryan Schmidt, Tovi Grossman, George Fitzmaurice, and Björn Hartmann. 2014. A series of tubes. In Proceedings of the 27th annual ACM symposium on User interface software and technology - UIST '14. ACM Press, New York, New York, USA, 3-12. https://doi.org/10.1145/2642918.2647374

[50] Martin Schmitz, Martin Herbers, Niloofar Dezfuli, Sebastian Günther, and Max Mühlhäuser. 2018. Off-Line Sensing. In Proceedings of the 2018 CHI Conference on Human Factors in Computing Systems - CHI '18, Vol. 2018-April. ACM Press, New York, New York, USA, 1-8. https://doi.org/10.1145/3173574.3173756

[51] Martin Schmitz, Andreas Leister, Niloofar Dezfuli, Jan Riemann, Florian Müller, and Max Mühlhäuser. 2016. Liquido. In Proceedings of the 2016 CHI Conference Extended Abstracts on Human Factors in Computing Systems - CHI EA '16. ACM Press, New York, New York, USA, 2688-2696. https://doi.org/10.1145/2851581. 2892275

[52] American Chemical Society. 2016. Guidelines for Chemical Laboratory Safety in Secondary Schools. (2016).

[53] Gabor Soter, Martin Garrad, Andrew T. Conn, Helmut Hauser, and Jonathan Rossiter. 2019. Skinflow: A soft robotic skin based on fluidic transmission. In 2019 2nd IEEE International Conference on Soft Robotics (RoboSoft). IEEE, 355-360. https://doi.org/10.1109/ROBOSOFT.2019.8722744

[54] Kenjiro Takemura, Shinichi Yokota, Mamoru Suzuki, Kazuya Edamura, Hideo Kumagai, and Tsunehiko Imamura. 2009. A liquid rate gyroscope using electroconjugate fluid. Sensors and Actuators A: Physical 149, 2 (Feb. 2009), 173-179. https://doi.org/10.1016/J.SNA.2008.12.004

[55] Yutaka Tokuda, Jose Luis Berna Moya, Gianluca Memoli, Timothy Neate, Deepak Ranjan Sahoo, Simon Robinson, Jennifer Pearson, Matt Jones, and Sriram Subramanian. 2017. Programmable Liquid Matter. In Proceedings of the Interactive Surfaces and Spaces on ZZZ - ISS '17. ACM Press, New York, New York, USA, 142-150. https://doi.org/10.1145/3132272.3134132

[56] Zhiliang Wan, Hongjun Zeng, and Alan Feinerman. 2006. Area-tunable micromirror based on electrowetting actuation of liquid-metal droplets. Applied Physics Letters 89, 20 (Nov. 2006), 201107. https://doi.org/10.1063/1.2388943

[57] Michael Wehner, Ryan L. Truby, Daniel J. Fitzgerald, Bobak Mosadegh, George M. Whitesides, Jennifer A. Lewis, and Robert J. Wood. 2016. An integrated design and fabrication strategy for entirely soft, autonomous robots. Nature 536, 7617 (Aug. 2016), 451-455. https://doi.org/10.1038/nature19100

[58] James P. Wissman, Kaushik Sampath, Alec Ikei, Kadri B. Özütemiz, Carmel Majidi, and Charles A. Rohde. 2019. Soft-matter pressure sensors for turbulence detection. In Sensors and Smart Structures Technologies for Civil, Mechanical, and Aerospace Systems 2019, Kon-Well Wang, Hoon Sohn, Haiying Huang, and Jerome P. Lynch (Eds.). SPIE, 82. https://doi.org/10.1117/12.2514358

[59] Qin Xu, Nikolai Oudalov, Qiti Guo, Heinrich M Jaeger, and Eric Brown. 2012. Effect of oxidation on the mechanical properties of liquid gallium and eutectic gallium-indium. Physics of fluids 24, 6 (2012), 063101.

[60] Hongjun Zeng, Zhiliang Wan, and Alan D. Feinerman. 2006. Tilting Micromirror With a Liquid-Metal Pivot. Fournal of Microelectromechanical Systems 15, 6 (Dec. 2006), 1568-1575. https://doi.org/10.1109/JMEMS.2006.883531 